Research Article

\title{
The Number of Central Lymph Nodes on Preoperative Ultrasound Predicts Central Neck Lymph Node Metastasis in Papillary Thyroid Carcinoma: A Prospective Cohort Study
}

\author{
Luying Gao $\mathbb{D}^{1},{ }^{1}$ Juanjuan Wang, ${ }^{1}$ Yuxin Jiang, ${ }^{1}$ Qiong Gao, ${ }^{1}$ Ying Wang, ${ }^{1}$ Xuehua Xi, ${ }^{2}$ \\ and Bo Zhang $\mathbb{1}^{2}$ \\ ${ }^{1}$ Department of Ultrasound, Chinese Academy of Medical Sciences \& Peking Union Medical College Hospital, Beijing, China \\ ${ }^{2}$ Department of Ultrasound, China-Japan Friendship Hospital, Beijing, China \\ Correspondence should be addressed to Bo Zhang; thyroidus@163.com
}

Received 8 August 2019; Revised 20 February 2020; Accepted 18 March 2020; Published 14 April 2020

Academic Editor: Franco Veglio

Copyright (c) 2020 Luying Gao et al. This is an open access article distributed under the Creative Commons Attribution License, which permits unrestricted use, distribution, and reproduction in any medium, provided the original work is properly cited.

\begin{abstract}
To evaluate the effectiveness of the number of central compartment lymph nodes (CLNs) on ultrasound (US) in predicting CLN metastasis (CLNM). We prospectively studied 309 papillary thyroid cancer (PTC) patients who underwent thyroidectomy with CLN dissection at our center from May 2017 to July 2017. The number and features of CLNs were evaluated preoperatively via US. All US examinations were performed using a Philips iU 22 or a GE Logiq 9 machine. Correlations between CLNs observed via preoperative US and amount of CLNM were evaluated. We found that $\geq 2$ CLNs on the preoperative US was associated with CLNM $(P<0.01)$. For this feature, the sensitivity, specificity, and area under the curve (AUC) were 54.3\%, 66.1\%, and 0.61, respectively. The presence of both suspected metastasis and $\geq 2$ CLNs on US had a specificity of $86.5 \%$. In addition, $\geq 3$ CLNs on preoperative US was associated with large-volume CLNM ( $>5$ metastatic CLNs) $(P<0.01)$. For this feature, the sensitivity, specificity and AUC were $54.8 \%, 74.5 \%$ and 0.65 , respectively. The presence of both suspected metastasis and $\geq 3$ CLNs on US had a specificity of $84.9 \%$. The presence of suspected metastasis and/or $\geq 3$ CLNs had a sensitivity of $80.6 \%$. Our results suggest that $\geq 2$ and $\geq 3$ CLNs on preoperative US may serve as ancillary preoperative markers for predicting CLNM.
\end{abstract}

\section{Introduction}

Papillary thyroid cancer (PTC) accounts for $85 \%$ of the cases of differentiated thyroid cancer. Despite a generally benign course of PTC, early cervical lymph node (CLN) metastasis may occur. Twenty percent to $60 \%$ of PTC patients have LN involvement, with malignant LNs more frequently located in the central compartment [1-9]. PTC patients with LN involvement have a poorer prognosis than those without LN involvement [10]. Large-volume LN metastasis (LNM) is defined as the presence of more than five metastatic LNs and has been shown to be associated with a $20 \%$ risk of local recurrence but also an increased risk of distant metastasis [11, 12].

Ultrasonography is the preferred screening modality for preoperative assessment of regional LNs in PTC patients [13]. However, the deep location of central compartment LNs (CLNs) renders it difficult to delineate these LNs on ultrasound (US). Preoperative neck US has a low sensitivity (44.4\%) for detecting CLN metastasis (CLNM) [14]. A recent trend toward routine central LN dissection avoiding radioactive treatment is still debated [15-17], and prophylactic central neck dissection (pCND) in patients with clinically uninvolved $\mathrm{cN} 0$ has been ineffective in some studies and is accompanied by postoperative complications $[18,19]$. Thus, finding preoperative features to predict CLNM is crucial, particularly large-volume CLNM, for PTC patients. This prospective study sought to assess the value of the number of CLNs on US for predicting CLNM and large-volume CLNM in patients with PTC.

\section{Patients and Methods}

2.1. Patients and Definitions. We prospectively studied 501 adult ( $>18$ years) patients with pathologically proven PTC 
who underwent thyroidectomy at our center between May 2017 and July 2017. Two experienced radiologists at our center evaluated preoperative US for CLNs. We included patients who underwent total or nearly total thyroidectomy with CLN dissection $(n=309)$ (Figure 1).

Hashimoto's thyroiditis was determined pathologically. Extracapsular invasion included both microscopic and gross invasion by the tumour. Based on the number of metastatic central LNs, patients were divided into three groups according to CLNM status: no CLNM, small-volume CLNM ( $\leq 5$ metastatic CLNs), or large-volume LNM ( $>5$ metastatic CLNs) $[11,12]$.

2.2. Thyroid US Examination. All US examinations were performed using a Philips iU 22 machine (Philips Healthcare, Eindhoven, Netherlands) or a GE Logiq 9 machine (GE Healthcare, Milwaukee, WI, USA); all US machines were equipped with a $5-12 \mathrm{MHz}$ linear-array transducer. The ultrasonographic examinations included central cervical LNs and the size of the thyroid nodules. In cases involving multifocal PTC, the largest thyroid nodule on US was recorded. The number, size, shape, and structure of LNs, cystic appearance, hyperechogenic punctuations, hyperechogenic hilum, and vascularity, were evaluated by US. Suspicious sonographic features of central cervical LNs included enlargement (short axis $>5 \mathrm{~mm}$ ), loss of the fatty hilum, a rounded rather than oval shape, hyperechogenicity, cystic change, calcifications, and peripheral vascularity. LNs were considered suspicious when 1 or more of the suspicious US findings were present [1].

US images were prospectively evaluated by two radiologists who were experienced in thyroid US and were blinded to patient clinical data and pathological results. Discrepancy between the two assessments was resolved by consensus after discussion.

The study protocol was approved by the Ethics Committee of our hospital.

2.3. Postoperative Complications. Major postoperative complications were recorded including neck hematoma requiring surveillance, transient or permanent unilateral recurrent nerve injury, and transient or permanent hypoparathyroidism. Serum calcium levels were assayed based on the evolution of clinical and biochemical parameters. In the case of hypocalcaemia, parathyroid hormone (PTH) levels were determined. Hypoparathyroidism was considered permanent if it lasted more than 6 months and required medical therapy with normal serum PTH levels. Paralysis of the recurrent laryngeal nerve was confirmed by laryngoscopy and considered permanent if it persisted for more than 6 months.

2.4. Statistical Analysis. Quantitative data were presented as means \pm standard deviations (SDs). Qualitative data were presented as frequencies. A Shapiro-Wilk test was used to evaluate whether data were normally distributed. Betweengroup differences for nonparametric data were analysed using a Mann-Whitney $U$ test. Parametric data were analysed using an unpaired $t$-test to evaluate between-group differences. A chi-square test with Yates' correction and Fisher's exact test were used to compare categorical variables. Sensitivities, specificities, positive predictive values (PPVs), negative predictive values (NPVs), and accuracies were calculated by comparing pathological findings. Receiver operating characteristic (ROC) curve analyses were used to calculate optimal cut-off values. A logistic regression model was used to evaluate risk factors. $P<0.05$ was considered statistically significant. Statistical analyses were performed using SPSS software (version 19.0, SPSS Inc., Chicago, IL, USA).

\section{Results}

3.1. Demographic and Baseline Features of the Patients. The demographic and baseline features of the patients are listed in Table 1. Among the 309 patients, 171 (55.3\%) patients had no CLNM, 107 (34.6\%) patients had smallvolume CLNM, and 31 (10.0\%) patients had large-volume CLNM. The mean age of the patients was $43.6 \pm 10.6$ years. The majority of the patients were female $(n=234 ; 75.7 \%)$. The mean size of the primary tumour was $1.0 \pm 0.6 \mathrm{~cm}$, and $113(36.2 \%)$ cases involved a tumour $>1 \mathrm{~cm}$. We examined all case records and identified the following postoperative complications: hematoma in surgical bed in 13 cases (4.2\%), permanent recurrent laryngeal nerve injury in 2 cases $(0.6 \%$; laryngoscopy showing paralysis persisting beyond 6 months), and permanent hypoparathyroidism requiring replacement therapy in 2 cases $(0.6 \%)$.

3.2. Clinical and US Features and Status of Central Lymph Node Metastases. We analysed the correlation between features and CLNM. The younger group of patients (age $<55$ years old) tended to have a high prevalence of CLNM compared to older patients $(P<0.01)$. Relative to a smaller tumour, a larger tumour $(>1.0 \mathrm{~cm})$ was significantly associated with CLNM $(P<0.01)$. The presence of suspected metastatic CLNs on preoperative US was significantly associated with CLNM $(P<0.01)$. Extracapsular invasion was also significantly associated with CLNM $(P<0.01)$. We further analysed the correlation between features and the number of CLNMs. Larger tumour size $(>1.0 \mathrm{~cm})$ was significantly associated with large-volume CLNM $(P<0.01)$. The presence of suspected metastatic CLNs on preoperative US was significantly associated with large-volume CLNM $(P<0.01)$ (Table 1).

Table 2 shows the sensitivity, specificity, positive predictive value (PPV), negative predictive value (NPV), accuracy, area under the curve (AUC) of younger age $(<55$ years), larger tumour size $(>1.0 \mathrm{~cm})$, suspected metastatic CLNs on preoperative US, and extracapsular invasion for CLNM. Table 3 shows the sensitivity, specificity, positive predictive value (PPV), negative predictive value (NPV), accuracy, and area under the curve (AUC) of larger tumour size $(>1.0 \mathrm{~cm})$ and suspected metastatic CLNs on preoperative US for large-volume CLNM. 


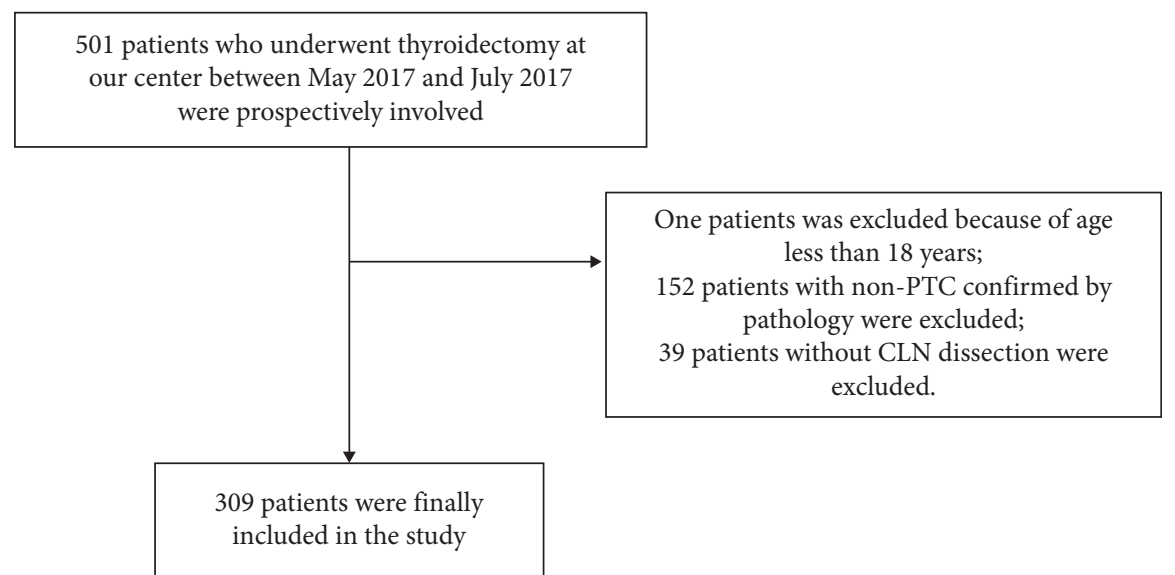

FIGURE 1: The flow chart of selection of papillary thyroid carcinoma patients.

TABle 1: The demographic and baseline features and status of central lymph node metastases in 309 patients with PTC.

\begin{tabular}{|c|c|c|c|c|}
\hline & No CLNM & Small-volume CLNM & Large-volume CLNM & $P$ \\
\hline Age & & & & $<0.01$ \\
\hline$<55$ & $131(49.8 \%)$ & $103(39.2 \%)$ & $29(11.0 \%)$ & \\
\hline$\geq 55$ & $40(88.9 \%)$ & $4(8.9 \%)$ & $2(4.4 \%)$ & \\
\hline Sex & & & & 0.29 \\
\hline Male & $36(48.0 \%)$ & $29(38.7 \%)$ & $10(13.3 \%)$ & \\
\hline Female & $135(57.7 \%)$ & $78(33.3 \%)$ & $21(8.9 \%)$ & \\
\hline Size of primary tumour & & & & $<0.01$ \\
\hline$\leq 1 \mathrm{~cm}$ & $123(62.8 \%)$ & $60(30.6 \%)$ & $13(6.6 \%)$ & \\
\hline$>1 \mathrm{~cm}$ & $48(42.5 \%)$ & $47(41.6 \%)$ & $18(15.9 \%)$ & \\
\hline Hashimoto's thyroiditis & & & & 0.72 \\
\hline Yes & $44(54.3 \%)$ & $27(33.3 \%)$ & $10(12.3 \%)$ & \\
\hline No & $127(55.7 \%)$ & $80(35.1 \%)$ & $21(9.2 \%)$ & \\
\hline Presence of CLNs on US & & & & 0.05 \\
\hline Yes & $111(52.6 \%)$ & $73(34.6 \%)$ & $27(12.8 \%)$ & \\
\hline No & $60(60.6 \%)$ & $34(34.3 \%)$ & $4(4.0 \%)$ & \\
\hline Suspected metastatic CLNs on US & & & & $<0.01$ \\
\hline Yes & $41(37.6 \%)$ & $46(42.2 \%)$ & $22(20.2 \%)$ & \\
\hline No & $130(65.0 \%)$ & $61(30.5 \%)$ & $9(4.5 \%)$ & \\
\hline
\end{tabular}

CLNM, central compartment lymph node metastasi. CLN, central compartment lymph nodes. US, ultrasound.

TABle 2: Diagnostic efficiency of features for CLNM status.

\begin{tabular}{|c|c|c|c|c|c|c|}
\hline & Sensitivity (\%) & Specificity (\%) & PPV (\%) & NPV (\%) & Accuracy $(\%)$ & AUC \\
\hline Age $<55$ years & 95.7 & 23.4 & 50.2 & 87.0 & 55.7 & 0.59 \\
\hline Tumour size $>1.0 \mathrm{~cm}$ & 47.1 & 71.9 & 57.5 & 62.8 & 60.8 & 0.60 \\
\hline$\geq 2$ CLNs & 54.3 & 66.1 & 56.4 & 64.2 & 60.8 & 0.61 \\
\hline Suspected metastatic CLNs & 49.3 & 76.0 & 62.4 & 65.0 & 64.1 & 0.63 \\
\hline Suspected metastasis and $\geq 2$ CLNs & 40.6 & 86.5 & 70.9 & 64.3 & 66.0 & 0.64 \\
\hline Suspected metastasis and/or $\geq 2$ CLNs & 63.0 & 55.6 & 53.4 & 65.1 & 58.9 & 0.59 \\
\hline
\end{tabular}

CLNM, central compartment lymph node metastasis. CLN, central compartment lymph nodes. AUC, area under the curve. PPV, positive predictive value. NPV, negative predictive value.

3.3. Risk Factors for CLNM and Large-Volume CLNM. To identify independent factors associated with CLNM and large-volume CLNM, significant variables were entered into the final models for multivariate testing. Suspected metastatic CLN on preoperative US was an independent risk factor for CLNM (odds ratio $[\mathrm{OR}]=1.64$ (95\%confidence interval (95\% CI) 1.23-2.19), $P<0.01)$, and suspected metastatic CLN on preoperative US was an independent risk factor for large-volume CLNM $(\mathrm{OR}=1.96 \quad(95 \% \quad \mathrm{CI}$ $1.25-3.05), P=0.003)$. Tumour size $(>1 \mathrm{~cm})$ was an independent risk factor for CLNM $(\mathrm{OR}=2.24 \quad(95 \%$ CI $1.33-3.77), P=0.002)$. Younger age ( $<55$ years) was another independent risk factor for CLNM $(\mathrm{OR}=8.00 \quad(95 \% \mathrm{CI}$ 3.13-20.41), $P<0.01)$. 
TABle 3: Diagnostic efficiency of features for large-volume CLNM.

\begin{tabular}{|c|c|c|c|c|c|c|}
\hline & Sensitivity (\%) & Specificity (\%) & PPV (\%) & NPV $(\%)$ & Accuracy $(\%)$ & AUC \\
\hline Tumour size $>1.0 \mathrm{~cm}$ & 58.1 & 65.8 & 15.9 & 93.4 & 65.0 & 0.62 \\
\hline$\geq 3$ CLNs & 54.8 & 74.5 & 19.3 & 93.7 & 72.4 & 0.65 \\
\hline Suspected metastatic CLNs on preoperative US & 71.0 & 68.7 & 20.2 & 95.5 & 68.9 & 0.70 \\
\hline Suspected metastasis and $\geq 3$ CLNs & 45.2 & 84.9 & 25.0 & 93.3 & 80.9 & 0.65 \\
\hline Suspected metastasis and/or $\geq 3$ CLNs & 80.6 & 58.3 & 17.7 & 96.4 & 60.5 & 0.70 \\
\hline
\end{tabular}

CLNM, central compartment lymph node metastasis. CLN, central compartment lymph nodes. AUC, area under the curve. PPV, positive predictive value. NPV, negative predictive value.

Table 4 displays the sensitivity and specificity of each suspected metastatic US signs (enlargement, loss of the fatty hilum, a rounded rather than oval shape, hyperechogenicity, cystic change, calcifications, and peripheral vascularity) for CLNM.

3.4. The Number of CLNs on Preoperative US and Status of Central Lymph Node Metastases. The number of CLNs on preoperative US was significantly associated with CLNM $(P<0.01)$. ROC curves demonstrated that 2 was the best cutoff value for CLNs on preoperative US. The sensitivity, specificity, PPV, NPV, accuracy, and AUC for $\geq 2$ visible CLNs were $54.3 \%, 66.1 \%, 56.4 \%, 64.2 \%, 60.8 \%$, and 0.61 (95\% CI 0.55-0.67), respectively. We further assessed the added diagnostic value provided by the criteria of $\geq 2$ CLNs with respect to suspected metastatic CLNs. The presence of both suspected metastasis and $\geq 2$ CLNs on US was significantly associated with CLNM $(P<0.01)$. The sensitivity, specificity, PPV, NPV, accuracy, and AUC were $40.6 \%$, $86.5 \%, 70.9 \%, 64.3 \%, 66.0 \%$, and 0.64 (95\% CI, 0.57-0.70), respectively. The presence of suspected metastasis and/or $\geq 2$ CLNs was also significantly associated with CLNM $(P<0.01)$. The sensitivity, specificity, PPV, NPV, accuracy, and AUC were $63.0 \%, 55.6 \%, 53.4 \%, 65.1 \%, 58.9 \%$, and 0.59 (95\% CI, 0.53-0.66), respectively (Table 2).

The number of CLNs on preoperative US was significantly associated with large-volume CLNM $(P<0.01)$. ROC curves demonstrated that 3 was the best cut-off value for CLNs on preoperative US. The sensitivity, specificity, PPV, NPV, accuracy, and AUC for $\geq 3$ CLNs were 54.8\%, 74.5\%, $19.3 \%, 93.9 \%, 72.4 \%$, and 0.65 (95\% CI, 0.54-0.75), respectively. We further assessed the added diagnostic value provided by the criteria of $\geq 3$ CLNs with respect to suspected metastatic CLNs. The presence of both suspected metastasis and $\geq 3$ CLNs on US was significantly associated with largevolume CLNM $(P<0.01)$. The sensitivity, specificity, PPV, NPV, accuracy, and AUC were 45.2\%, 84.9\%, 25.0\%, 93.3\%, $80.9 \%$, and 0.65 (95\% CI, 0.54-0.76), respectively. The presence of suspected metastasis and/or $\geq 3$ CLNs was also significantly associated with CLNM $(P<0.01)$. The sensitivity, specificity, PPV, NPV, accuracy, and AUC were $80.6 \%, 58.3 \%, 17.7 \%, 96.4 \%, 60.5 \%$, and 0.70 (95\% CI, $0.60-0.79$ ), respectively (Table 3 ).

3.5. The Diagnostic Value of CLN Number for PTC Patients with Hashimoto's Thyroiditis. We calculated the diagnostic value of the number of CLNs for those PTC patients combined with Hashimoto's thyroiditis $(N=63) . \geq 2$ CLNs on preoperative US was significantly associated with CLNM $(P=0.02)$. The sensitivity, specificity, accuracy, and AUC for $\geq 2$ CLNs were $81.8 \%, 43.3 \%, 63.5 \%$, and 0.63 (95\% CI, $0.49-0.77)$, respectively.

We calculated the diagnostic value of the number of CLNs for those PTC patients combined without Hashimoto's thyroiditis $(N=246)$. $\geq 2$ CLNs on preoperative US was significantly associated with CLNM $(P<0.01)$. The sensitivity, specificity, accuracy, and AUC for $\geq 2$ CLNs were $45.9 \%$, $71.5 \%, 60.2 \%$ and 0.59 (95\% CI, 0.52-0.67), respectively. $\geq 3$ CLNs on preoperative US was significantly associated with large-volume CLNM $(P<0.01)$. The sensitivity, specificity, accuracy, and AUC for $\geq 3$ CLNs were $50.0 \%, 81.7 \%, 78.0 \%$, and 0.67 (95\% CI, 0.55-0.79), respectively.

\section{Discussion}

In our investigation, younger age, larger tumour size, extracapsular invasion, and suspected metastatic CLNs on preoperative US were associated with CLNM of PTC patients. Furthermore, we found that $\geq 2$ CLNs was associated with CLNM, and $\geq 3$ CLNs was associated with large-volume CLNM. For predicting CLNM, the presence of suspected metastasis and/or $\geq 2$ CLNs had a relative high sensitivity. For predicting large-volume CLNM, the presence of both suspected metastasis and $\geq 3$ CLNs had a relative high specificity, and the presence of suspected metastasis and/or $\geq 3$ CLNs had a relative high sensitivity. In our study, Hashimoto's thyroiditis was not associated with CLNM, and for patients with Hashimoto's thyroiditis, the diagnostic value of the number of CLNs is still positive.

Due to the location of CLNs, it is difficult to identify their features via US. As a result, preoperative US exhibits low sensitivity for detecting CLNM [14]. In our study, $49.3 \%$ of the PTC patients with CLNM had suspected metastatic CLNs on preoperative US; $20.2 \%$ of the PTC patients with suspected metastatic CLNs had large-volume CLNM based on pathological findings. These results show that the accuracy of preoperative US for evaluating CLNs is unreliable. Since our study is a prospective study, the number and US feature of LNs are of great concern when examining the CLNs. Our findings demonstrate that $\geq 2$ CLNs and $\geq 3$ CLNs are valuable preoperative clinical parameters for predicting CLNM or large-volume CLNM. Moreover, our findings suggest that the number of CLNs on US provides added diagnostic value to that of suspected metastasis observed in CLNs via US. The high specificity and PPV afforded by 
TABLE 4: The diagnostic efficiency of suspected metastatic ultrasound signs.

\begin{tabular}{|c|c|c|c|c|}
\hline Signs & Sensitivity, (\%) & Specificity, (\%) & No CLNM, (\%) & CLNM, (\%) \\
\hline Enlargement & 16.2 & 95.2 & $8(25.8)$ & $23(74.2)$ \\
\hline Loss of the fatty hilum & 45.8 & 79.0 & $35(35.0)$ & $65(65.0)$ \\
\hline Round shape & 16.2 & 91.0 & $15(49.5)$ & $23(60.5)$ \\
\hline Microcalcifications or hyperechogenicity & 12.0 & 97.0 & $5(22.7)$ & $17(77.3)$ \\
\hline Cystic aspect & 11.3 & 100.0 & $0(0.0)$ & $16(100.0)$ \\
\hline Peripheral vascularity & 14.8 & 98.2 & $3(12.5)$ & $21(87.5)$ \\
\hline
\end{tabular}

CLNM, central compartment lymph node metastasis.

presentation of both suspected metastasis and $\geq 2$ CLNs could help rule in CLNM if the outcome is positive. The high sensitivity and NPV afforded by presentation of suspected metastasis and/or $\geq 3$ CLNs may help rule out large-volume CLNM if the outcome is negative. These findings suggest that treatment strategies should be modified for patients with a relatively large number of CLNs and suspected metastatic CLNs on preoperative US. Since large-volume pathologic lymph node metastasis is an important recurrence factor by ATA risk stratification system [1] and more than half of the patients operated on showed no CLNM, $\geq 3$ CLNs on preoperative US may serve as a cut-off marker for prophylactic central node dissection of patients with PTC. Moreover, the results showed that $\geq 2$ or $\geq 3$ CLNs on preoperative US were not an independent risk factor for predicting CLNM and may serve as an ancillary marker with respect to suspected metastatic CLNs.

Age at diagnosis of thyroid cancer is identified as an independent predictor of disease-specific survival in most published staging systems $[20,21]$. A previous study showed that large-volume CLNM was more frequently observed in younger patients [19]. This phenomenon was also demonstrated by a recent international multicentre retrospective study in which the age cut-off was shifted from 45 to 55 years [22]. Our results are comparable to these previously reported findings. Younger age is a valuable parameter for detecting CLNM.

Tumour size was significantly associated with LN metastasis in patients with PTC. Kim et al. found that compared with patients with smaller tumours, patients with larger tumours had a higher rate of postoperative recurrence, invasion, and CLN metastasis [23, 24]. Our results, which are comparable to those reported in previous studies, showed that larger tumour size $(\geq 1 \mathrm{~cm})$ was correlated with CLNM and large-volume CLNM. Our findings suggest that age and tumour size may serve as preoperative ancillary markers for determining the extent of surgery.

Hashimoto's thyroiditis patients often have central lymph node enlargement [24]. In our study, Hashimoto's thyroiditis was not associated with CLNM. Moreover, we calculated the diagnostic value of the number of CLNs for those PTC patients combined with Hashimoto's thyroiditis. $\geq 2$ CLNs on preoperative US was significantly associated with CLNM for those PTC patients combined with Hashimoto's thyroiditis. For predicting CLNM, the sensitivity, specificity, accuracy, and AUC for $\geq 2$ CLNs were $81.8 \%, 43.3 \%, 63.5 \%$, and 0.63 , respectively. It seems that in PTC patients with Hashimoto's thyroiditis, the diagnostic value of the number of CLNs declines, but is still meaningful.
The 2015 ATA guideline suggests active surveillance for nodules $<1 \mathrm{~cm}$ in the absence of evidence of extrathyroidal extension, metastatic cervical lymph nodes, or distant metastases [1]. However, the treatment of micropapillary thyroid cancer is still controversial. In clinical practice, still a large number of nodules $<1 \mathrm{~cm}$ received FNA or surgical resection. There are several reasons for these patients with thyroid malignant nodules $<1 \mathrm{~cm}$ to select FNA and surgery. In China, patients have poor compliance with active followup. During the follow-up, patients need to receive imaging examinations, blood tests, etc., but the medical insurance system does not cover the examinations in the outpatient clinic. Due to time costs, labor costs, and other expense issues, doctors and patients in China may be more inclined to choose surgery. Another important reason is more than $30 \%$ cervical lymph node metastasis in CNO PTC and extrathyroidal extension $[25,26]$. With the application of ACR-TIRADS and ATA guidelines in recent years, this situation has been improving [27].

Our study has several limitations. First, all the patients underwent thyroidectomy, which may have led to selection bias resulting in underestimation of NPV and overestimation of PPV for preoperative US, younger age and larger tumour size. Second, our study included 501 patients, only 31 of whom had large-volume CLNM. Future studies that involve more subjects may produce more accurate results.

\section{Conclusion}

The criteria of $\geq 2$ CLNs and $\geq 3$ CLNs on preoperative US have effective diagnostic value for predicting CLNM and large-volume CLNM in patients with PTC. The number of CLNs on preoperative US may provide additional information for predicting CLNM. In PTC patients with Hashimoto's thyroiditis, the diagnostic value of the number of CLNs decreases, but is still valuable. Our results suggest that treatment may be modified for PTC patients with a relatively large number of CLNs and suspected metastatic CLNs on preoperative US.

\section{Data Availability}

The data used to support the findings of this study are available from the corresponding author upon request.

\section{Ethical Approval}

Institutional review board approval was obtained from our center for this study. 


\section{Consent}

Informed consent was obtained for publication of patient data.

\section{Conflicts of Interest}

The authors declare that they have no conflicts of interest.

\section{Acknowledgments}

This study was supported by the National Natural Science Foundation of China (No. 81541131) and the International Science and Technology Cooperation Program of China (2015DFA30440).

\section{References}

[1] B. R. Haugen, E. K. Alexander, K. C. Bible et al., "American thyroid association management guidelines for adult patients with thyroid nodules and differentiated thyroid cancer: the American thyroid association guidelines task force on thyroid nodules and differentiated thyroid cancer," Thyroid, vol. 26, no. 1, pp. 1-133, 2015.

[2] S. K. G. Grebe and I. D. Hay, "Thyroid cancer nodal metastases: biologic significance and therapeutic considerations," Surgical Oncology Clinics of North America, vol. 5, no. 1, pp. 43-63, 1996.

[3] G. F. W. Scheumann, O. Gimm, G. Wegener, H. Hundeshagen, and H. Dralle, "Prognostic significance and surgical management of locoregional lymph node metastases in papillary thyroid cancer," World Journal of Surgery, vol. 18, no. 4, pp. 559-567, 1994.

[4] Y. Ito, T. Uruno, K. Nakano et al., "An observation trial without surgical treatment in patients with papillary microcarcinoma of the thyroid," Thyroid, vol. 13, no. 4, pp. 381-387, 2003.

[5] S.-M. Chow, S. C. K. Law, J. K. C. Chan, S.-K. Au, S. Yau, and W.-H. Lau, "Papillary microcarcinoma of the thyroid?Prognostic significance of lymph node metastasis and multifocality," Cancer, vol. 98, no. 1, pp. 31-40, 2003.

[6] M. A. Kouvaraki, S. E. Shapiro, B. D. Fornage et al., "Role of preoperative ultrasonography in the surgical management of patients with thyroid cancer," Surgery, vol. 134, no. 6, pp. 946-954, 2003.

[7] S. Lebolleux, E. Girard, M. Rose et al., "Ultrasound criteria of malignancy for cervical lymph nodes in patients followed up for differentiated thyroid cancer," Journal of Clinical Endocrinology and Metabolism, vol. 92, no. 9, pp. 3590-3594, 2007.

[8] A. Frasoldati and R. Valcavi, "Challenges in neck ultrasonography: lymphadenopathy and parathyroid glands," Endocrine Practice, vol. 10, no. 3, pp. 261-268, 2004.

[9] S. K. Kuna, I. Bracic, V. Tesic, K. Kuna, G. H. Herceg, and D. Dodig, "Ultrasonographic differentiation of benign from malignant neck lymphadenopathy in thyroid cancer," Journal of Ultrasound in Medicine, vol. 25, no. 12, pp. 1531-1537, 2006.

[10] T. S. Wang, S. Dubner, L. A. Sznyter, and K. S. Heller, "Incidence of metastatic well-differentiated thyroid cancer in cervical lymph nodes," Archives of Otolaryngology-Head \& Neck Surgery, vol. 130, no. 1, pp. 110-113, 2004.

[11] G. W. Randolph, Q.-Y. Duh, K. S. Heller et al., "The prognostic significance of nodal metastases from papillary thyroid carcinoma can Be stratified based on the size and number of metastatic lymph nodes, as well as the presence of extranodal extension," Thyroid, vol. 22, no. 11, pp. 1144-1152, 2012.

[12] A. Machens and H. Dralle, "Correlation between the number of lymph node metastases and lung metastasis in papillary thyroid cancer," The Journal of Clinical Endocrinology \& Metabolism, vol. 97, no. 12, pp. 4375-4382, 2012.

[13] P. R. Larsen, "New guidelines for patients with thyroid nodules and differentiated thyroid cancer," Nature Clinical Practice Endocrinology \& Metabolism, vol. 2, no. 6, p. 297, 2006.

[14] M. T. Khokhar, K. M. Day, R. B. Sangal et al., "Preoperative high-resolution ultrasound for the assessment of malignant central compartment lymph nodes in papillary thyroid cancer," Thyroid, vol. 25, no. 12, pp. 1351-1354, 2015.

[15] C. Gambardella, E. Tartaglia, A. Nunziata et al., "Clinical significance of prophylactic central compartment neck dissection in the treatment of clinically node-negative papillary thyroid cancer patients," World Journal of Surgical Oncology, vol. 14, no. 1, p. 247, 2016.

[16] G. Conzo, C. Mauriello, G. Docimo et al., "Clinicopathological pattern of lymph node recurrence of papillary thyroid cancer. Implications for surgery," International Journal of Surgery, vol. 12, no. Suppl 1, pp. S194-S197, 2014.

[17] C. Gambardella, R. Patrone, F. Di Capua et al., "The role of prophylactic central compartment lymph node dissection in elderly patients with differentiated thyroid cancer: a multicentric study," BMC Surgery, vol. 18, no. s1, p. 110, 2019.

[18] L. Chen, Y.-H. Wu, C.-H. Lee, H.-A. Chen, E.-W. Loh, and K.-W. Tam, "Prophylactic central neck dissection for papillary thyroid carcinoma with clinically uninvolved central neck lymph nodes: a systematic review and meta-analysis," World Journal of Surgery, vol. 42, no. 9, pp. 2846-2857, 2018.

[19] H.-S. Oh, S. Park, M. Kim et al., "Young age and male sex are predictors of large-volume central neck lymph node metastasis in clinical N0 papillary thyroid microcarcinomas," Thyroid, vol. 27, no. 10, pp. 1285-1290, 2017.

[20] S. Leboulleux, E. Girard, M. Rose et al., "Ultrasound criteria of malignancy for cervical lymph nodes in patients followed up for differentiated thyroid cancer," The Journal of Clinical Endocrinology \& Metabolism, vol. 92, no. 9, pp. 3590-3594, 2007.

[21] I. Ganly, I. J. Nixon, L. Y. Wang et al., "Survival from differentiated thyroid cancer: what has age got to do with it?" Thyroid, vol. 25, no. 10, pp. 1106-1114, 2015.

[22] I. J. Nixon, D. Kuk, V. Wreesmann et al., "Defining a valid age cutoff in staging of well-differentiated thyroid cancer," Annals of Surgical Oncology, vol. 23, no. 2, pp. 410-415, 2016.

[23] K.-M. Kim, J.-B. Park, K.-S. Bae, and S.-J. Kang, "Analysis of prognostic factors in patients with multiple recurrences of papillary thyroid carcinoma," Surgical Oncology, vol. 21, no. 3, pp. 185-190, 2012.

[24] S. S. Kim, B.-J. Lee, J.-C. Lee et al., "Preoperative ultrasonographic tumor characteristics as a predictive factor of tumor stage in papillary thyroid carcinoma," Head \& Neck, vol. 33, no. 12, pp. 1719-1726, 2011.

[25] L. Zhang, J. Yang, Q. Sun et al., "Risk factors for lymph node metstasis in papillary thyroid microcarcinoma: older patients with fewer lymph node metastases," European Journal of Surgical Oncology (EJSO), vol. 42, no. 10, pp. 1478-1482, 2016.

[26] D. Y. Lee, S. M. Hwang, J. H. An et al., "Predicting extrathyroidal extension in patients with papillary thyroid microcarcinoma according to a BRAF mutation," Clinical and Experimental Otorhinolaryngology, vol. 10, no. 2, pp. 174-181, 2017.

[27] F. N. Tessler, W. D. Middleton, E. G. Grant et al., "ACR thyroid imaging, reporting and data system (TI-RADS): white paper of the ACR TI-RADS committee," Journal of the American College of Radiology, vol. 14, no. 5, pp. 587-595, 2017. 